\title{
Trace Metals in Groundwater \& Vadose Zone Calcite: In Situ Containment \& Stabilization of Stronthium-90 \& Other Divalent Metals \& Radionuclides at Arid West DOE
}

\author{
Robert W. Smith \\ Subsurface Science \\ University of Idaho - Idaho Falls \\ 1776 Science Center Drive \\ Idaho Falls, ID 83402
}

\section{RESEARCH OBJECTIVES}

Radionuclide and metal contaminants such as strontium-90 are present beneath U.S. Department of Energy (DOE) lands in both the groundwater (e.g., 100-N area at Hanford, WA) and vadose zone (e.g., Idaho Nuclear Technology and Engineering Center at the Idaho National Engineering and Environmental Laboratory). In situ containment and stabilization of these contaminants is a cost-effective treatment strategy. However, implementing in situ containment and stabilization approaches requires definition of the mechanisms that control contaminant sequestration. We are investigating the in situ immobilization of radionuclides or contaminant metals (e.g., strontium-90) by their facilitated co-precipitation with calcium carbonate in groundwater and vadose zone systems. Our facilitated approach, shown schematically in Figure 1, relies upon the hydrolysis of introduced urea to cause the acceleration of calcium carbonate precipitation (and trace metal co-precipitation) by increasing $\mathrm{pH}$ and alkalinity. Subsurface urea hydrolysis is catalyzed by the urease enzyme, which may be either introduced with the urea or produced in situ by ubiquitous subsurface urea hydrolyzing microorganisms. Because the precipitation process tends to be irreversible and many western aquifers are saturated with respect to calcite, the co-precipitated metals and radionuclides will be effectively removed from the aqueous phase over the long-term. Another advantage of the ureolysis approach is that the ammonium ions produced by the reaction can exchange with radionuclides sorbed to subsurface minerals, thereby enhancing the availability of the radionuclides for recapture in a more stable solid phase (co-precipitation rather than adsorption). Our specific research objectives include: * Elucidation of the mechanisms and rates for the release of sorbed trace metals and their subsequent sequestration by co-precipitation in calcite induced by urea hydrolysis. * Evaluation at the field scale of the influence of microbial calcite precipitation on the partitioning and retention of strontium and other naturally occurring divalent metals in groundwater. * Identification of specific microbial characteristics that signify subsurface geochemical conditions conducive to ureolytically-driven calcite precipitation.

\section{RESEARCH PROGRESS AND IMPLICATIONS}

The objectives are being accomplished by conducting integrated field and laboratory research evaluating (a) the precipitation rate of calcite through in situ experiments and (b) the partitioning of strontium and other metals into carbonate minerals through laboratory experiments and field characterization. Key results to date include: * Demonstrating in laboratory and field the linkage between urea hydrolysis and calcite precipitation. * Observing that strontium is incorporated into calcite precipitated by urea hydrolyzers, with higher distribution coefficients than in abiotic systems. * Determining that urea hydrolyzers are ubiquitous in the SRPA. * Developing and applying PCR primers specific to bacterial urease subunit C. These results are summarized below. Demonstrating in laboratory and field setting the linkage between urea hydrolysis and calcite precipitation. Laboratory experiments using pure cultures of Bacillus pasteurii, a known urea hydrolyzer, have yielded kinetic expressions that couple the hydrolysis of urea with the precipitation of calcite over a temperature range $(10$ to $20 \mathrm{C}$ ) relevant to groundwater conditions. These results showed a 10 fold increase in the first-order rate constant for urea hydrolysis over the temperature range investigated. However, no temperature dependence was observed over the range of investigation for the rate constant of the second-order chemical affinity based expression describing calcite precipitation. Calcite precipitation via urea hydrolysis has also been demonstrated in a field setting. In a single-well push pull test conducted in the Snake River Plain aquifer, the injection of low levels of molasses (7.5 ppm) increased the in situ urea 
hydrolysis rate by a factor of 270 . Subsequent injection of mmolar levels of urea resulted in the precipitation of calcite. Preliminary analysis of these field results suggest that hydrolysis rate are slower, but the same order as observed in laboratory experiments. Detailed analysis of this experiment is an ongoing activity. Observing that $\mathrm{Sr}$ is incorporated into calcite precipitated by urea hydrolyzers, with higher distribution coefficients than in abiotic systems. Batch laboratory experiments using B. pasteurii yielded distributions coefficients for strontium in calcite that were significantly higher than those obtained for ammonium carbonate controls. Time-of-Flight Secondary Ion Mass Spectrometry and X-ray absorption spectroscopy analyses were both consistent with the presence of strontium in the calcite as a solid solution. Our results suggest that observed differences between the B. pasteurii precipitated calcites and calcites precipitated in the control experiments are the result of differences in the precipitation rate. Developing and applying PCR primers specific to bacterial urease subunit C. Urease-specific polymerase chain reaction (PCR) primers designed to amplify ureC, a gene that codes for a conserved subunit of the urease enzyme, were developed to detect aquifer microbes with the ability to hydrolyze urea. Several sets of PCR oligonucleotide primers were designed based on an alignment and phylogenetic analysis of known ureC genes. The developed primers were used to determine the presence of urease genes in complex microbial communities collected from the Snake River Plain Aquifer before, during, and after the groundwater had been stimulated using molasses and urea using a single-well push-pull test. The PCR data suggest that following urea and molasses addition the urease gene target was detected more consistently and in greater abundance. The molecular tools are important for demonstrating that microbially catalyzed urea hydrolysis (and associated calcite precipitation) is occurring in situ. The goal of ongoing research activity is to determine that ureC genes are actually being expressed (presence of mRNA that is specific to urease) as a way to confirm the activity of urease positive cells.

\section{PLANNED ACTIVITIES}

We plan to continue the development and application of molecular level tools to evaluate ureolytic activity of subsurface microbial communities. Our goal is to have a set of methods developed and ready for application in the single well push-pull experiments described below. Biotic and abiotic experiments will also be conducted to develop coupled chemical reaction/transport models. These experiments will differ from previous experiments primarily through the inclusion of aquifer minerals and the consideration of solution advection. In addition, a static multilevel sampler experiment will be conducted in a Snake River Plain Aquifer well in winter and spring of 2004. We expect to have a useable coupled model by summer of 2004. This model will be used to design a series of new single-well push-pull field experiments at a site analogous to the INEEL that will be initiated in summer of 2004. These field experiments will be used to evaluate the effectiveness of alternative electron donor/urea amendment approaches in precipitating calcites and sequestering strontium and other metals. Finally, we will actively seek out opportunities to apply our research results at a contaminated DOE site. Specifically, we plan to apply our microbial techniques to water samples and aseptic cores collected from Handford 100-N area. 